\title{
Some Workout Problems on Motion of Raindrop
}

\author{
Sneha Dey ${ }^{1,2}$ and A. Ghorai ${ }^{2}$ \\ ${ }^{1}$ Physics Department, Maulana Azad College \\ Kolkata, India \\ Email: sneha072000 [AT] gmail.com \\ ${ }^{2}$ School of Science, Jangalpur Road \\ Kolkata, India \\ Email: amitavaghorai61 [AT] gmail.com
}

\begin{abstract}
The motion of rain drop through atmosphere is an interesting classical problem because of the fact that air resistance and moisture accretion are integral part of it. Mathematical modeling of it using Newtonian formalism is considered here and discussions are made for no mass accretion and air resistance proportional to nth power of velocity. We use python program and library extensively to find the terminal velocity of rain drop. Graphs show close agreement and velocity power up to $n=3$ is good.
\end{abstract}

Keywords - Rain drop, terminal velocity, air resistance, mass accretion.

\section{INTRODUCTION}

The major component of the water cycle is rain, responsible for depositing most of the fresh water on the earth and the major cause of rain production is moisture. Precipitation falls for enough moisture and upward motion of convective clouds (those with strong upward vertical motion such as cumulonimbus or thunder clouds) which can organize into narrow rain bands [1]. When upslope flow is maximized within windward sides of the terrain, heavy precipitation in mountainous areas is possible. On the leeward side of mountains, desert climates can exist due to the dry air caused by down slope flow which causes heating and drying of the air mass. Down slope flow causes heating and drying of the air mass on the leeward side of mountains and so desert climates can exist due to the dry air.

\section{PHYSICAL PROPERTIES}

Raindrops differ widely in their shapes, sizes and velocities. Smaller raindrops are generally spherical in shape. However, as size of the drop increases, it becomes an oblate spheroid. The raindrop is axially symmetric along the line of motion and in general non-spherical. The shape will be governed by internal hydrostatic pressure, hydrodynamic pressure of medium and surface tension. Beard and Chuang [2] describe the shape of a raindrop as a 10th order cosine distortion of a sphere as

$$
\mathrm{r}(\theta)=\mathrm{R}\left(1+\sum_{\mathrm{i}=1}^{10} \mathrm{c}_{\mathrm{n}} \cos n \theta\right)
$$

Here $\mathrm{R}$ is the radius of the undistorted sphere in meter, $\mathrm{c}_{\mathrm{n}}$ 's are the coefficients that depend on the radius of the drop and $\theta$ is the polar angle of elevation.

Raindrops have a wide size distribution. A commonly used empirical distribution for rain drop size is the Marshall Palmer distribution [3] N(a) $=8 \times 10^{6} \mathrm{e}^{-8200 \mathrm{Rh}^{-0.21}}$

Here $\mathrm{h}$ is the rain rate given in $\mathrm{mm} / \mathrm{hr}$ and $\mathrm{N}(\mathrm{a})$ is the number of rain drops per unit volume that contains sizes within the interval $(R, R+d R)$. Large drops are severely distorted, while smaller drops are almost spherical. Note that the drops that make up a significant fraction of rain are less than $1 \mathrm{~mm}$ in size and are not severely distorted and their shapes can be well approximated by a sphere. Therefore, in this paper, we will model rain drops as transparent spheres of water.

As a raindrop falls, it attains a constant velocity, called the terminal velocity [4]. Gunn and Kinzer [5] present an empirical study of the terminal velocities of falling raindrops for different drop sizes. Their observations show that the terminal velocity of a raindrop can be expressed as a function of its size and is given by

$$
\mathrm{v}_{\mathrm{T}}=\sqrt{\frac{4 \mathrm{~g}(2 \mathrm{R})\left(\rho_{\mathrm{w}}-\rho_{\mathrm{a}}\right)}{\mathrm{b} \rho_{\mathrm{a}}}}
$$

Here $\mathrm{v}_{\mathrm{T}}$ is in (meter/sec) and $\mathrm{R}$ is in meters. Here The first order differential equation of rain drop considering nth power of velocity for air resistance is solved for the first time by both analytically as well as using the library 
of python code for no mass accretion which is based on the undergraduate theoretical knowledge. Emphasis is given so that undergraduate students can think of novel ideas; increase their skill of understanding; to create new method for proper understanding, etc.

\section{THE PROBLEM}

In general this kind of problem can be solved using time integral theorem which states that the time integral of force or impulse is equal to the integral of linear momentum. Mathematically we can write it as $\int \overrightarrow{\mathrm{dp}}=\int \overrightarrow{\mathrm{F}} \mathrm{dt}$.

Let at time $t$ the mass of a raindrop is $m$ and velocity $\vec{v}$ and those at a later time $t+d t$ are $m+d m$ and $\vec{v}+\overrightarrow{d v}$ respectively. Thus

$$
\overrightarrow{\mathrm{F}} \mathrm{dt}=(\mathrm{m}+\mathrm{dm})(\overrightarrow{\mathrm{v}}+\overrightarrow{\mathrm{dv}})-\mathrm{m} \overrightarrow{\mathrm{v}}
$$

Or,

$$
\overrightarrow{\mathrm{F}}=\mathrm{m} \frac{\overrightarrow{\mathrm{dv}}}{\mathrm{dt}}+\frac{\mathrm{dm}}{\mathrm{dt}} \overrightarrow{\mathrm{v}}
$$

In this case the motion will be governed by the weight of the raindrop mgk̂k, buoyant force $\frac{m \rho_{a} g}{\rho_{w}} \widehat{k}$, and frictional force of the medium, which is proportional to the nth power of velocity $b^{n} \hat{k}$ where $\rho_{a}$ and $\rho_{w}$ are the density of the medium and raindrop respectively. We assume $\mathrm{a}=\mathrm{g}\left(1-\frac{\rho_{\mathrm{a}}}{\rho_{\mathrm{w}}}\right)$ and then

$$
\mathrm{m} \frac{\overrightarrow{\mathrm{dv}}}{\mathrm{dt}}+\frac{\mathrm{dm}}{\mathrm{dt}} \overrightarrow{\mathrm{v}}=\mathrm{am} \hat{\mathrm{k}}-\mathrm{bv}^{\mathrm{n}} \hat{\mathrm{k}}
$$

Here $b$ is the resistive force per unit nth power of velocity. Lynch and Lommatsch [6] took the value of resistive constant as $b=0.15 \rho_{\mathrm{a}} \mathrm{A}$ where the cross sectional area of raindrop assumes the form $\mathrm{A}=3.3108 \times(2 \mathrm{R})^{2.21672}$, with mass of raindrop as $\mathrm{m}=957.251 \times(2 \mathrm{R})^{3.09275}$ where $\mathrm{R}$ is the approximate radius. Generally we put $\mathrm{n}=1$ for resistive force proportional to velocity. But there are examples where $n>1$ and generalized procedure for solution is to be taken in those cases.

\section{NO MASS VARIATION CASE}

In this no mass variation case since $\frac{\mathrm{dm}}{\mathrm{dt}}=0$ we get from equation (5)

$$
\frac{d v}{\mathrm{am}-v^{\mathrm{n}}}=\frac{\mathrm{bdt}}{\mathrm{m}}
$$

When $\mathrm{n}=0$ we have $\mathrm{v}=\mathrm{a}-\frac{\mathrm{bt}}{\mathrm{m}}$ and for $\frac{\mathrm{dv}}{\mathrm{dt}}=0$ we have any value of the terminal velocity including zero because am $=$ $\mathrm{b}$ and thus clearly $\mathrm{am} \geq \mathrm{b}$.

For $\mathrm{n}=1$ we have $\mathrm{v}=\frac{\mathrm{am}}{\mathrm{b}}\left(1-\mathrm{e}^{-\frac{\mathrm{bt}}{\mathrm{m}}}\right)$ and terminal velocity is $\mathrm{v}_{\mathrm{T}}=\frac{\mathrm{am}}{\mathrm{b}}$.

When $\mathrm{n}=2$ the roots of $\frac{\mathrm{am}}{\mathrm{b}}-\mathrm{v}^{2}$ are $\pm \sqrt{\frac{\mathrm{am}}{\mathrm{b}}}$ and the solution is $\mathrm{v}=\sqrt{\frac{\mathrm{am}}{\mathrm{b}}} \frac{1-\mathrm{e}^{-\mathrm{t} \sqrt{\frac{4 \mathrm{ab}}{\mathrm{m}}}}}{1+\mathrm{e}^{-\mathrm{t} \sqrt{\frac{4 \mathrm{ab}}{\mathrm{m}}}}} \mathrm{v}=\sqrt{\frac{a m}{b} 1-e^{-t \sqrt{\frac{4 a b}{m}}}}$. Thus the terminal velocity can be obtained for $\mathrm{t} \rightarrow \infty$ as $\mathrm{v}_{\mathrm{T}}=\sqrt{\frac{\mathrm{am}}{\mathrm{b}}}$.

When $n=3$ the roots of $\frac{a m}{b}-v^{3}$ are $\left(\frac{a m}{b}\right)^{\frac{1}{3}}, \frac{-1 \pm i \sqrt{3}}{2}\left(\frac{a m}{b}\right)^{\frac{1}{3}}$. Neglecting negative and imaginary terms as they have no physical meaning the solution is $\mathrm{v}=\left(\frac{\mathrm{am}}{\mathrm{b}}\right)^{\frac{1}{3}}\left[1-e^{-\left(\frac{a m}{b}\right)^{\frac{2}{3}} \frac{3 b t}{m}}\right]$. The terminal velocity can be obtained for $\mathrm{t} \rightarrow \infty$ as $\mathrm{v}_{\mathrm{T}}=$ $\left(\frac{\mathrm{am}}{\mathrm{b}}\right)^{\frac{1}{3}}$.

When $\mathrm{n}=4$ the roots of $\frac{\mathrm{am}}{\mathrm{b}}-\mathrm{v}^{4}$ are $\pm\left(\frac{\mathrm{am}}{\mathrm{b}}\right)^{\frac{1}{4}}, \pm \mathrm{i}\left(\frac{\mathrm{am}}{\mathrm{b}}\right)^{\frac{1}{4}}$. Hence $\mathrm{v}=\left(\frac{\mathrm{am}}{\mathrm{b}}\right)^{\frac{1}{4}}\left[1-e^{-\frac{b t}{m}}\right]^{\frac{1}{4}}$. The terminal velocity for $\mathrm{t} \rightarrow \infty$ as $\mathrm{v}_{\mathrm{T}}=\left(\frac{\mathrm{am}}{\mathrm{b}}\right)^{\frac{1}{4}}$.

Using the procedure of rational fraction of integration the first order differential equation for nth power of velocity reduces to

$$
\int_{0}^{v} \sum_{l=1}^{n} \frac{s_{1} d v}{r_{1}-v}=\int_{0}^{t} \frac{b d t}{m} \Rightarrow e^{\frac{b t}{m}}=\prod_{l=1}^{n}\left(\frac{r_{l}}{r_{1}-v}\right)^{s_{l}}
$$

We can use another general procedure to solve equation (6). For that, we put $x=1-\frac{\mathrm{bv}^{\mathrm{n}}}{\mathrm{am}}$ and take $p=\frac{\mathrm{n}-1}{\mathrm{n}}$. So $d x=$ $-\frac{\mathrm{bnv}^{\mathrm{n}-1} \mathrm{dv}}{\mathrm{am}}$ and thus the equation reduces to $\frac{\mathrm{Amdx}}{\mathrm{bnxv}^{\mathrm{n}-1}}=\mathrm{adt}$

Or,

$$
-\frac{b n d t}{m}=\frac{d x}{x\left[\frac{a m}{b}(1-x)\right]^{\frac{n-1}{n}}}=\frac{d x}{x}\left[\frac{a m}{b}(1-x)\right]^{-p}=\left(\frac{a m}{b}\right)^{-p} \frac{d x}{x}\left[1+p x+\frac{p(p+1)}{2 !} x^{2}+\frac{p(p+1)(p+2)}{3 !} x^{3}+\cdots\right]
$$


Or,

$$
-\left(\frac{\mathrm{am}}{\mathrm{b}}\right)^{\mathrm{p}} \frac{\mathrm{bndt}}{\mathrm{m}}=\frac{\mathrm{dx}}{\mathrm{x}} \sum_{\mathrm{l}=0}^{\infty} \frac{(\mathrm{p}-1+\mathrm{l}) !}{(\mathrm{p}-1) ! ! !} \mathrm{x}^{\mathrm{l}} \Rightarrow-\left(\frac{\mathrm{am}}{\mathrm{b}}\right)^{\mathrm{p}} \frac{\mathrm{bn}(\mathrm{t}+\mathrm{C})}{\mathrm{m}}=\sum_{\mathrm{l}=0}^{\infty} \frac{(\mathrm{p}-1+1) ! \mathrm{x}^{1}}{(\mathrm{p}-1) ! ! !}=\sum_{\mathrm{l}=0}^{\infty} \frac{\left(1-\frac{1}{\mathrm{n}}\right) !}{1\left(-\frac{1}{n}\right) ! ! !}\left(1-\frac{\mathrm{bv}^{\mathrm{n}}}{\mathrm{am}}\right)^{1}
$$

This is obtained by integration. Now at $\mathrm{t}=0$ we have $\mathrm{v}=0$ and $\mathrm{C}=-\frac{\mathrm{m}}{\mathrm{bn}}\left(\frac{\mathrm{b}}{\mathrm{am}}\right)^{\frac{1-\mathrm{n}}{\mathrm{n}}} \sum_{\mathrm{l}=0}^{\infty} \frac{\left(1-\frac{1}{\mathrm{n}}\right) !}{1\left(-\frac{1}{n}\right) ! ! !}$. Thus

$$
-\left(\frac{\mathrm{am}}{\mathrm{b}}\right)^{\mathrm{p}} \frac{\mathrm{bnt}}{\mathrm{m}}=\sum_{\mathrm{l}=0}^{\infty} \frac{\left(1-\frac{1}{\mathrm{n}}\right) !}{1\left(-\frac{1}{\mathrm{n}}\right) ! ! !}\left(1-\frac{\mathrm{bv}^{\mathrm{n}}}{\mathrm{am}}\right)^{1}
$$

\section{DISCUSSIONS}

Table 1

\begin{tabular}{|c|c|c|c|}
\hline Air density $\rho_{a} \mathrm{~kg} / \mathrm{m}^{3}$ & $0.006211^{*}$ & Radius $R \mathrm{~m}$ & $0.0001-0.01$ \\
\hline Water density $\rho_{w} \mathrm{~kg} / \mathrm{m}^{3}$ & $957.251^{*}$ & $b=0.15 \rho_{a} A$ & $4.91 \times 10^{-12}-6.84 \times 10^{-5}$ \\
\hline $\begin{array}{c}\text { Acceleration due to gravity } \\
g \mathrm{~m} / \mathrm{s}^{2}\end{array}$ & $9.81^{*}$ & $a=g\left(1-\frac{\rho_{a}}{\rho_{w}}\right)$ & 9.8099363 \\
\hline
\end{tabular}

*ref [6]

For no mass accretion a python code is developed (matplotlib) and integration by odeint is (code is written in appendix) performed for different powers of velocity using the values of the parameters given in table 1 . The plot in logarithmic scale is shown in figure 1 where velocity reaches its terminal velocity almost within ten seconds for mass of rain drop $0.5 \mathrm{mg}$ and approximate diameter $1 \mathrm{~mm}$. As discussed in section 4 the velocity for $\mathrm{n}=0$ increases with time.

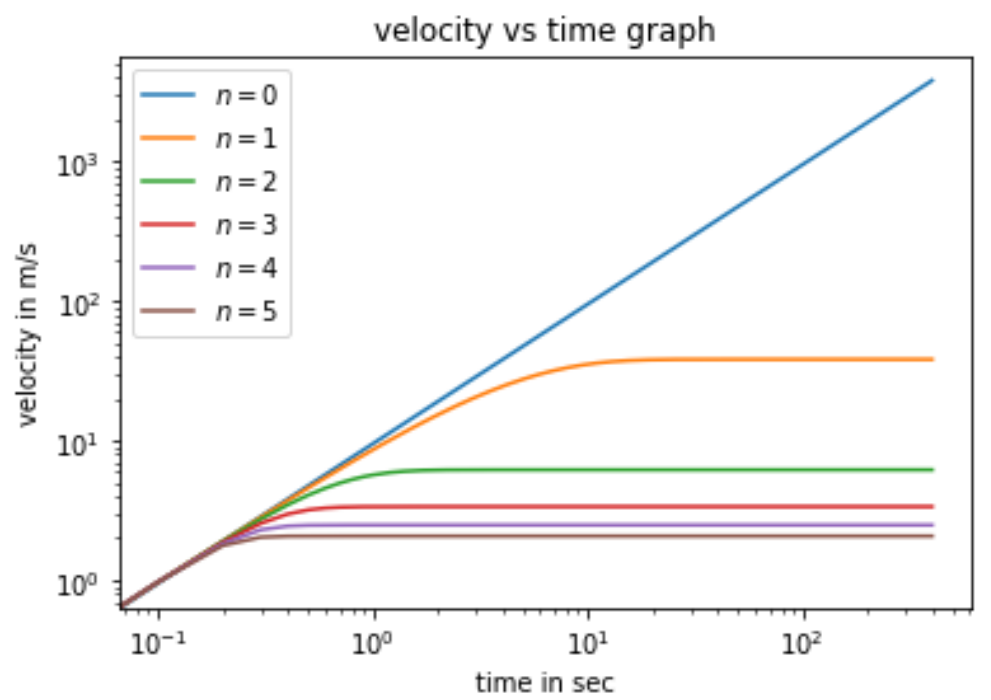

Figure 1

For further verification terminal velocity in logarithmic scale for $n=1,2,3,4,5$ is plotted against mass in logarithmic scale along with Gunn and Kinzer value [5]. This is shown in figure 2. The plots show close agreement and velocity power up to $n=3$ perhaps a better choice. Here $n=0$ is not plotted as the terminal velocity is undetermined.

The graphs contain a single intersecting point of approximate terminal velocity $1 \mathrm{~m} / \mathrm{s}$ and mass $10^{-06} \mathrm{mg}$. This perhaps is the choice of minimum raindrop size below which it may be assumed to be a cloud particle. We note that terminal velocity satisfies the equation $b v_{T}^{n}=$ ma which depends on mass of raindrop and value of $b$. Here again best agreement between analytical and graphical value of $v_{T}^{n}$ is observed.

\section{CONCLUSIONS}

These graphs clearly speak about the fact that diameter and mass have some impact on terminal velocity. Due to mass accretion in moist air the raindrop collects mass and rate may be $\frac{d m}{d t}=\rho_{a} v A$ where $A=\pi R^{2}$ is the projected area of the raindrop which is the largest cross section. For spherical drop, increase in radius is proportional to $m^{\frac{1}{3}}$ and an increase in area is proportional to $m^{\frac{2}{3}}$. Also mass accretion depends on velocity and so the raindrop size changes with time, i.e. $\mathrm{dm} / \mathrm{dt}$ is proportional to mass $\mathrm{m}$ and velocity $\mathrm{v}$. Thus $\frac{\mathrm{dm}}{\mathrm{dt}}=\mathrm{cm}^{\alpha} \mathrm{v}^{\beta}$ where $\mathrm{c}>0$ is a constant and $\alpha$ and $\beta$ are (almost) arbitrary 
exponents [7-9]. This form includes the two most commonly studied cases called the easy case $(\alpha, \beta)=\left(\frac{2}{3}, 0\right)$ and the hard case $(\alpha, \beta)=\left(\frac{2}{3}, 1\right)$. The constant $\mathrm{c}$ depends on actual shape of raindrop. If the raindrop is spherical then [10]

$$
c=\frac{\pi R^{2}}{\left(\frac{4}{3} \pi R^{3} \rho_{w}\right)^{\alpha}}
$$

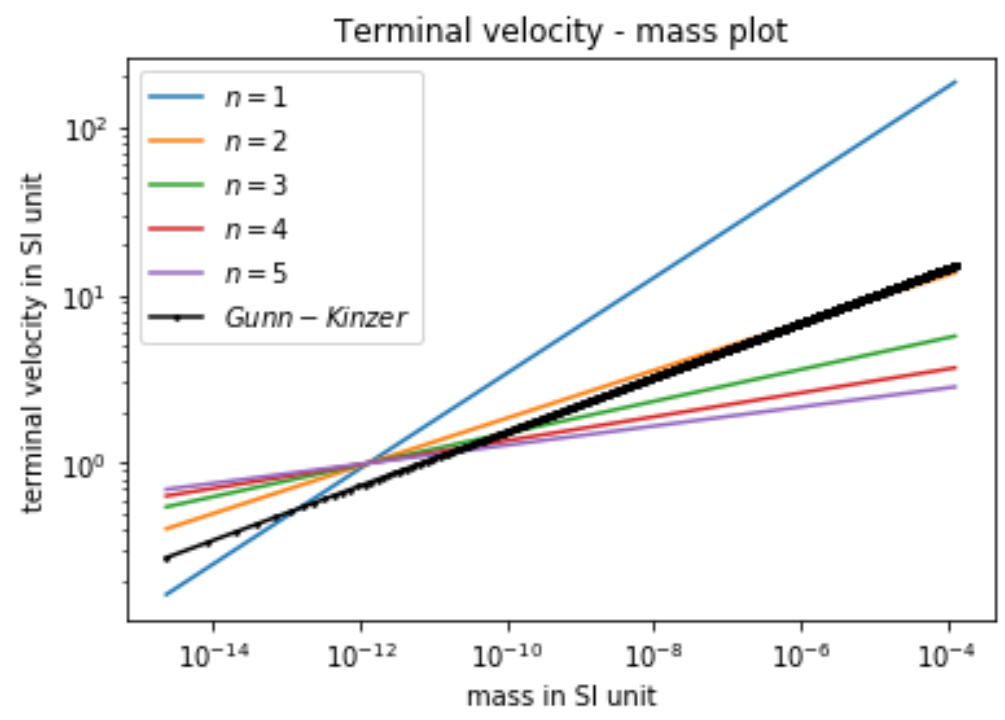

Figure 2

This modification seems inadequate because of pressure and temperature variations in the atmosphere. This is not considered in any literature so far.

\section{ACKNOWLEDGEMENT}

The first author did this whole work as online project fellow in covid situation under supervision of Dr. A. Ghorai at School of Science, Block 1/3G, 66/63 Jangalpur Road, Kolkata-81.

\section{REFERENCES}

[1] Kshitiz Garg and Shree K. Nayar, Photometric Model of a Rain Drop, www.semanticscholar.org @inproceedings 2003.

[2] K. V. Beard and C. Chaung. A new model for the equilibrium shape of raindrops. Journal of Atmospheric Science, vol. 44 no. (11), pp. 1509-1524, 1987.

[3] J. S. Marshall and W. M. K. Palmer. The distribution of raindrops with sizes. Journal of Meteorology, vol. 5, pp. 165166, 1948.

[4] R. M. Manning. Stochastic Electromagnetic Image Propagation. McGraw-Hill, Inc, 1993.

[5] R. Gunn and G. D. Kinzer. The terminal velocity for water droplet in stagnant air. Journal of Meteorology, vol. 6, pp. 243-248, 1949.

[6] Ben Lynch and Gavin Lommatsch, Modeling the velocity of a raindrop, May 6, 2011, http://home2.fvcc.edu/ dhicketh/DiffEqns/Spring11projects/Ben_Lynch_Gavin_Lom matsch/DiffEqProject/DiffEqProjectGavinBen.pdf [8 August 2012]

[7] K. S. Krane, American Journal of Physics vol. 49, pp. 113-117, 1981; doi: 10.1119/1.12537.

[8] I. Adawi, American Journal of Physics vol. 54, pp. 739-740, 1986; doi: 10.1119/1.14465.

[9] Alan D. Sokal1, American Journal of Physics vol. 78, pp. 643-645, 2010; doi: 10.1119/1.3246871.

[10] M. H. Partovi and D. R. Aston, American Journal of Physics vol. 57, pp. 912-920, 1989; doi: 10.1119/1.15846.

\section{APPENDIX}

PYTHON CODE

Program for $1^{\text {st }}$ graph :-

import numpy as np

from scipy.integrate import odeint

import matplotlib.pyplot as plt

$\mathrm{d}=10 * *(-3) * 1$

$\mathrm{c}=0.3$

$\mathrm{pw}=1000$ 


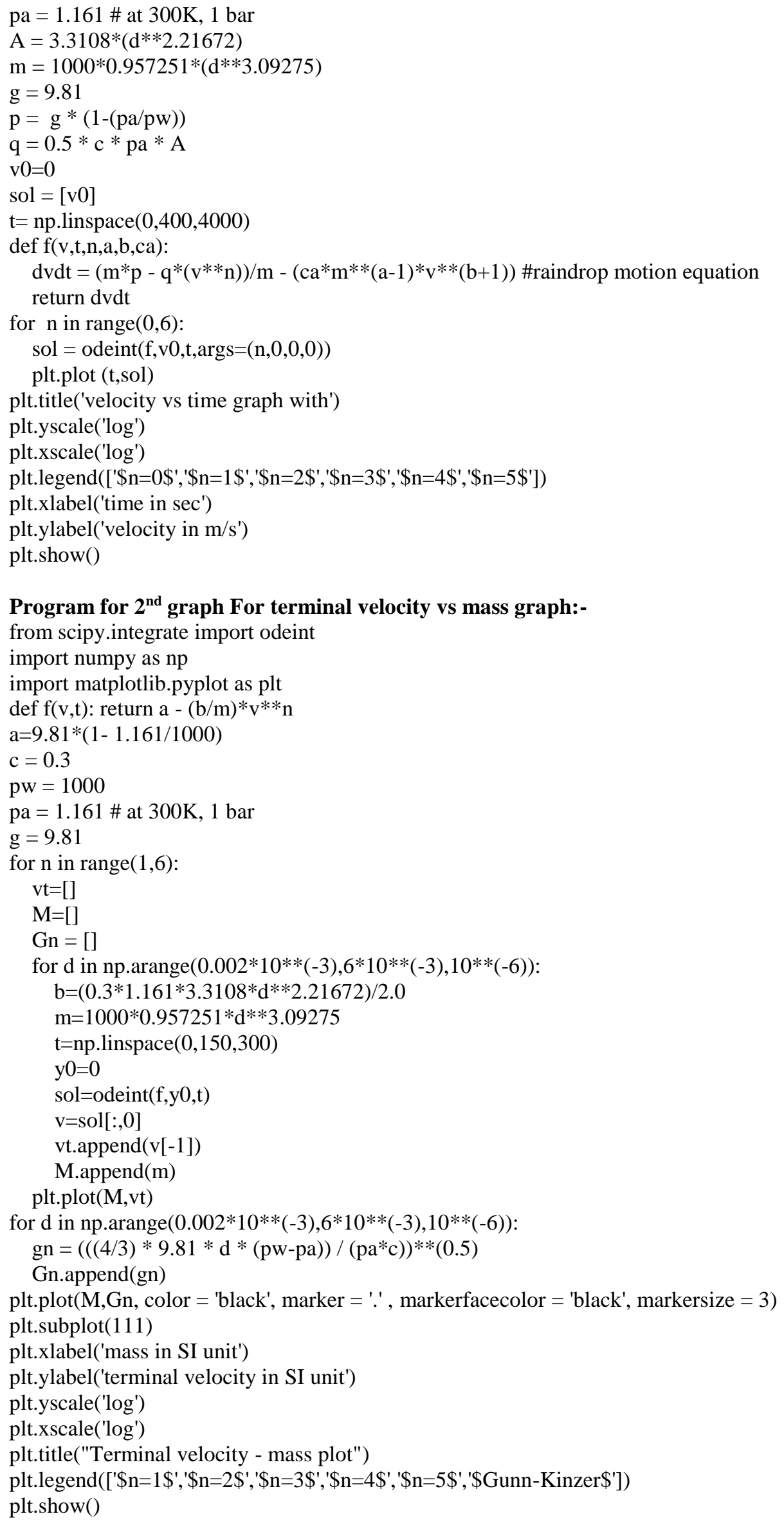

